\title{
Análisis comparativo de las prácticas educativas inclusivas en instituciones educativas de Santiago de Cali ${ }^{*}$
}

\section{Comparative analysis of inclusive educational practices in educational institutions of Santiago de Cali}

Claudia Patricia Rodríguez Andrade ${ }^{\star \star}$, Martha Liliana Urbano Burbano***
Recibido: 29-may-2019

Aceptado: 2-jul-2019

\section{Resumen}

El artículo presenta un análisis comparativo de las prácticas educativas inclusivas en Instituciones Educativas de Santiago de Cali. Para ello, se abordan posturas teóricas que brindan elementos para la inclusión y las prácticas de la educación inclusiva. Como metodología se utilizaron entrevistas semiestructuradas, encuestas cerradas, observación directa. Los hallazgos se asocian en categorías de análisis que se encaminan a una educación para todos y se relacionan con los aciertos que tiene cada una de las instituciones de acuerdo con lo que los estudiantes manifiestan al sentirse reconocidos.

\section{Palabras claves}

educación; prácticas; inclusión; reconocimiento.

\section{Abstract}

The article presents a comparative analysis of inclusive educational practices in educational institutions of Santiago de Cali. For this, theories that offer elements for inclusion and practices of inclusive education are approached. Semi-structured interviews, closed surveys and direct observation were used as methodology. The findings were associated in categories of analysis that are directed to an education for all and are related to the successes that each one of the institutions has had according to what students manifest when feeling recognized.

\section{Keywords}

education; practices; inclusion; recognition.

\footnotetext{
* El artículo es el resultado de la investigación Interfacultades sobre el análisis comparativo de las prácticas educativas inclusivas en insituciones de Santiago de Cali.

** Profesora e investigadora de la Fundación Universitaria Católica Lumen Gentium, magíster en Neuropsicología y Educación de la Universidad de la Rioja. Correo electrónico cprodriguez@unicatolica.edu.co Orcid: https://orcid.org/0000-0001-8067-2939

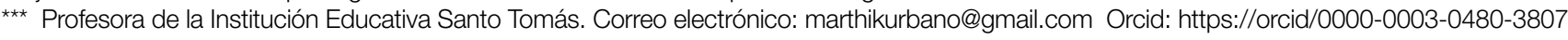




\section{Introducción}

Los procesos pedagógicos que se desarrollan en las Instituciones Educativas (IE) de Santiago de Cali evidencian diferentes prácticas inclusivas que se están aplicando actualmente en las aulas de clase para la construcción de los sujetos, dado que acuden estudiantes con características sociales, económicas, culturales y físicas, entre otras, que requieren espacios de aprendizaje propicios para la atención a la diversidad. En este sentido, se interroga sobre cómo las IE apropian el concepto de inclusión y adoptan las prácticas educativas inclusivas en sus contextos escolares.

Por lo expresado, la investigación abarca la inclusión y las prácticas educativas inclusivas de tres IE desde una vertiente teórica fundada a partir de la Declaración Universal de los Derechos Humanos como referente que consagra el derecho a la educación como "el pleno desarrollo de la personalidad humana y el fortalecimiento del respeto a los derechos humanos y a las libertades fundamentales" (Organización de las Naciones Unidas [ONU], 1948), que Rosa Blanco adopta al afirmar que "la educación es sin duda una poderosa herramienta para avanzar hacia sociedades más inclusivas y democráticas" (2014), al igual que Booth y Ainscow (2015), creadores de la guía para el índice de inclusión y promoción de valores y prácticas inclusivas, y finalmente, en Colombia con Wilmar
Ángel Ramírez, investigador sobre el tema. Desde este enfoque, participaron dos IE (en una de ellas participaron dos de sus sedes) seleccionadas aleatoriamente y de cada una de ellas, un coordinador, tres docentes y tres grupos de estudiantes.

La investigación pretende contribuir - sin el ánimo de ejercer valoración a ninguna de las instituciones- a los procesos de educación y a la vivencia escolar en pro de realizar una lectura que permita reflexionar sobre los retos y desafíos que se presentan, como también retomar acciones que fomenten las prácticas educativas inclusivas a partir de las visiones de los miembros de las comunidades educativas.

\section{Metodología}

La metodología implementada en esta investigación es mixta. El método utilizado es etnográfico y permitió analizar las prácticas docentes y evidenciar lo planeado, lo dicho y lo hecho en cuanto a los interrogantes que se aplicaron mediante los siguientes instrumentos: entrevistas semiestructuradas, encuestas cerradas y observación directa. La investigación estuvo compuesta por tres momentos: la aplicación de los instrumentos, la categorización y finalmente la interpretación y comparación de resultados. De esta manera, se identificaron coincidencias observadas y afirmadas por los miembros de la comunidad educativa, las cuales develan las realidades de la educación inclusiva.

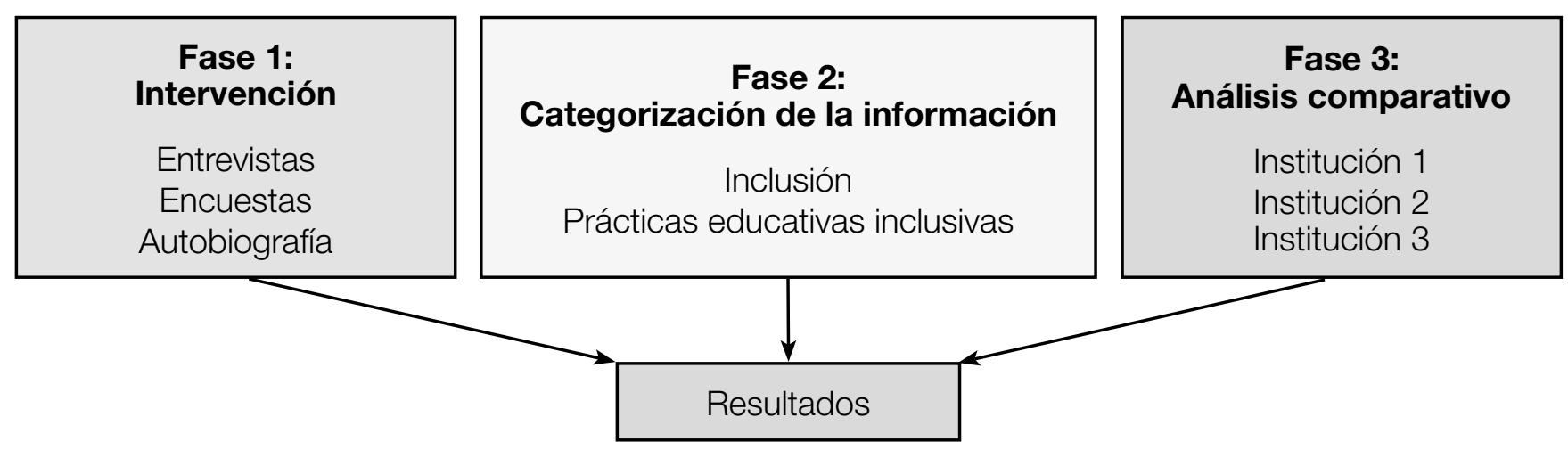

Figura 1: Fases de la investigación Fuente: elaboración propia 


\section{Aproximación institucional al concepto de inclusión}

La dinámica discursiva permitió indagar directamente sobre las percepciones que tienen los miembros de las Instituciones Educativas en este primer caso, con respecto a la intervención de las directivas en tanto orientadoras de maestros frente a lo que piensan sobre la inclusión en los contextos educativos. En este sentido, se identificó que todos los coordinadores tienen conocimiento de sus experiencias institucionales y reconocen elementos de la normatividad como eje esencial para guiar en su rol, pero cada uno de ellos muestra ausencias frente al conocimiento en profundidad de lo indagado de acuerdo con lo que mencionan ${ }^{1}$ :

[...] la ley de inclusión sale genéricamente, pero la divulgación no ha sido bien manejada por todo el mundo, considero que, bueno la inclusión no solo es la discapacidad, sino también de las víctimas del conflicto armado, de la gente que está en proceso de desplazamiento, en las chicas que están en situación de calle, por ejemplo, en las que tenemos aquí en el colegio, pero la verdad la gente no conoce los derechos frente a estas personas [...] (comunicación personal, coordinador 1, agosto 2018)

[...] durante este año que sacamos adelante el proyecto de inclusión desde una perspectiva más organizada con un plan de trabajo que incluyó también el conocimiento teórico legal de la inclusión educativa, en este caso, acercamiento al último decreto 1421 del 2017 [...] desde esa línea el apoyo de la Secretaría está muy interesado en fortalecer las prácticas inclusivas y por ende, también ofrece las capacitaciones a las que asistimos en representación del colegio [...] (comunicación personal, coordinador 2, agosto 2018)

1 Las transcripciones se realizaron de manera literal porque, aun cuando queden errores de sintaxis y elaboración de ideas, la forma en que se expresa cada uno puede ser materia de posteriores análisis y estudios.
[...] tengo claridad con respecto a lo más relevante, pues que todas las instituciones deben garantizar el acceso a los estudiantes con algún tipo de especial que se les debe prestar apoyo que se debe de hacer un seguimiento [...] no he tenido la oportunidad de participar con algún taller, me encantaría porque es un tema que me interesa mucho; tuve la oportunidad de trabajar instituciones que son especiales para niños con inclusión, por ejemplo la Fundación Ideal, tuve la oportunidad de hacer prácticas allá, pero en realidad que me haya capacitado, no. (comunicación personal, coordinador 3, septiembre 2018)

En lo expuesto se evidencia que el coordinador 1 expresa de manera amplia la inclusión como derecho y reconocimiento a la diversidad de los sujetos en el contexto, en este caso, el de cada uno de los estudiantes; a diferencia del coordinador 2, quien sustenta el concepto de inclusión desde la norma y da relevancia a los estudiantes con discapacidad y/o talentos excepcionales. Por otro lado, el tercer coordinador enfoca su conocimiento en la accesibilidad como elemento primordial en las instituciones, pero demuestra que no tiene claridad en todo y lo que sabe es desde la experiencia en otro lugar enfocado en la educación especial. De esta forma, los tres coordinadores se muestran acordes con lo que expresa Blanco (2014) al mencionar que la inclusión se suele asociar a los estudiantes con capacidad o necesidades educativas especiales y hacer referencia a que paulatinamente, el enfoque se está concibiendo de manera más amplia en pro de promover el acceso equitativo a una educación de calidad.

Por otro lado, con respecto a la percepción de los estudiantes se indagó frente a la forma cómo la inclusión está siendo recepcionada y reconocida a partir de elementos inclusivos como derecho y equidad en las Instituciones Educativas y en qué porcentaje de acuerdo con el muestreo y las entrevistas a profundidad. 
Tabla 1. La inclusión para los estudiantes

\begin{tabular}{cccc}
\hline Ítems & $\begin{array}{c}\text { Institución Educativa } \\
\text { Santa Isabel de Hungría } \\
\text { sede Alfonso López } \\
(\%)\end{array}$ & $\begin{array}{c}\text { Institución Educativa } \\
\text { Santa Isabel de Hungría } \\
\text { sede Llano Verde Invicali } \\
\text { (\%) }\end{array}$ & $\begin{array}{c}\text { Institución Educativa } \\
\text { República de Israel } \\
\text { (\%) }\end{array}$ \\
$\begin{array}{c}\text { En mi Institución me han } \\
\text { hablado sobre inclusión }\end{array}$ & 71 & 61 & 35 \\
$\begin{array}{c}\text { En mi Institución me siento } \\
\text { incluido en las actividades que } \\
\text { se realizan }\end{array}$ & 47 & 47 & 55 \\
$\begin{array}{c}\text { Comprendo que la inclusión } \\
\text { hace parte de los derechos que } \\
\text { tenemos todos los colombianos }\end{array}$ & 79 & 69 & 70 \\
\hline
\end{tabular}

\section{Fuente: elaboración propia}

De lo expuesto, se notifica que la inclusión, como medio para convivir en los diferentes contextos en igualdad de condiciones, convierte a las IE en el lugar en el que convergen de manera significativa la concientización de los sujetos y las formas de reconocimiento y participación en la sociedad, por ello, desde los procesos de formación se infunde el discurso, la compresión y el sentir del estudiante, y se demuestra que, aunque en las instituciones trabajadas los estudiantes reconocen que se les habla sobre inclusión, en un alto porcentaje se hace énfasis en esta como derecho y en otro sentido porcentualmente, aproximadamente la mitad siente que no hay equidad en el desarrollo de las actividades, ya que los estudiantes no siempre están siendo incluidos. De allí se divisa que independientemente de que se hable de inclusión y se la reconozca como derecho, falta avanzar en las acciones del maestro.

\section{Prácticas educativas inclusivas institucionales}

"Los valores son guías fundamentales y promueven la acción; nos impulsan hacia adelante, y nos dan un sentido de dirección definiendo un destino." (Booth y Ainscow, 2015)

En el marco de los valores, las prácticas educativas inclusivas institucionales desarrollan un conjunto de modos de accionar en los entornos, esto es en cierta medida, el fundamento que permite guiar y comprender lo que se debe realizar en los diversos contextos dado que vinculan las formas de hacer, relacionar e implementar frente a las diversas situaciones que pueden surgir en el medio. En este caso, se analiza a partir de las Instituciones Educativas y lo expresado por lo maestros, algunas de ellas se resaltan para ejemplificar las prácticas que están incidiendo en la vida escolar. Después se retoma un muestreo de los resultados en la encuesta y se mencionan aquellas prácticas observadas y verificadas por los miembros participantes de la comunidad educativa.

Desde un primer ejercicio realizado con los maestros en el primer contexto en la Institución Educativa República de Israel se evidenció el valor denominado por Booth y Ainscow (2015) como sabiduría, partiendo desde la inclusión de una de sus maestras (invidente), la cual expresó:

[...] El poder uno de compartir con las personas el poder uno el servir a la gente, compartir sus conocimientos, el poder uno como, por mi propio testimonio, de mostrarle a los estudiantes que sí se puede seguir adelante, que no hay impedimentos, que los impedimentos están en las aptitudes, en la mente, isí? [...] 
(comunicación personal, docente la, septiembre 2018)

En un segundo contexto, en la sede Alfonso López se identificó el valor de la esperanza a partir de una de las experiencias de un maestro en una jornada de descanso escolar:

[...] En el coliseo, que usted me vio a mi triste, aburrido y que yo le pregunté: ¿Por qué estás triste? y él me dijo: es que en el Icfes no me fue bien [...] y yo le dije es que el Icfes es el único camino para ser feliz y él me dijo: es que yo quería ingresar a la universidad [...] iy la universidad es el único camino para ser feliz? ¿Qué es lo que a vos te gusta hacer en la vida?, el a mí me respondió a mí me gustan es los negocios, entonces yo le dije: aproveche esa cualidad que usted tiene para hacer negocios, para comerciar, todo eso y si su papá tiene opciones dígale que le regale o le preste un capital y coloque el negocio [...] y el papá le colaboró, le abrió un Fruver [...] (comunicación personal, docente 2 c, octubre 2018)

En el tercer contexto, en la sede Llano Verde Invicali se observó la manera en que se ejemplifica el valor del respeto por la diversidad con la situación a continuación:

[...] La siguiente información que se brinda es referente al día miércoles, en el cual se celebrará el día de los niños, ante ello, la profesora hace una invitación a los estudiantes que deseen participar de un concurso que hay sobre la liga de la justicia y convoca a que los niños vengan disfrazados, usen un antifaz y si no, igual también pueden participar; hace énfasis en que cualquier niño puede venir y sin necesidad de venir disfrazado [...] (observación 3c, noviembre 2018)

Ante lo indicado, Parrilla y Susinos (2004), resaltan que al desarrollar acciones que han ido ganando terreno como movimiento, desafío y rechazo a las políticas, culturas y prácticas educativas, promueven cualquier tipo de exclusión. Esto es, en otras palabras, la forma en que las instituciones están transformando su mirada y desarrollando valores que conlleven adaptaciones, ajustes, mejoras que se van construyendo y brindan accesibilidad a los estudiantes, independientemente de sus características particulares. En este sentido, las tres instituciones manifestaron generar valores inclusivos en su comunidad y que mediante su fomento contribuyen a la formación de acciones incluyentes.

Desde otro punto de vista y con un muestreo significativo, en la encuesta los estudiantes presentaron los siguientes porcentajes:

Tabla 2. Acciones inclusivas para los estudiantes

\begin{tabular}{|c|c|c|c|}
\hline Ítems & $\begin{array}{l}\text { Institución Educativa } \\
\text { Santa Isabel de Hungría } \\
\text { sede Alfonso López (\%) }\end{array}$ & $\begin{array}{c}\text { Institución Educativa } \\
\text { Santa Isabel de Hungría } \\
\text { sede Llano Verde Invicali } \\
(\%)\end{array}$ & $\begin{array}{l}\text { Institución Educativa } \\
\text { República de Israel (\%) }\end{array}$ \\
\hline $\begin{array}{l}\text { En el salón de clase mi profesor } \\
\text { realiza actividades que nos ayudan } \\
\text { a comprender la situación física, } \\
\text { emocional o social que tengamos. }\end{array}$ & 40 & 62 & 72 \\
\hline $\begin{array}{l}\text { Mi profesor organiza espacios } \\
\text { adecuados en la clase en los } \\
\text { cuales todos podemos participar. }\end{array}$ & 43 & 55 & 94 \\
\hline $\begin{array}{l}\text { Mi profesor me explica en qué me } \\
\text { equivoco y me motiva para mejorar } \\
\text { de acuerdo con mis capacidades. }\end{array}$ & 35 & 68 & 82 \\
\hline
\end{tabular}


De lo anterior, se determina que los estudiantes continuamente se desenvuelven en diversas situaciones que permiten reconocer los factores a los que se enfrentan (académico, económico, social, cultural, político, entre otros), los cuales los llevan a estar en constantes acciones como prácticas inherentes a una calidad de vida. En este caso, los porcentajes para cada una de las instituciones frente a lo que el maestro hace, planea, explica y motiva se enmarcan, para menos de la mitad de los estudiantes de la Institución Educativa Santa Isabel de Hungría sede Alfonso López, en un promedio inferior, lo que deja entrever que, aunque se desarrollen las acciones, no alcanzan a ser comprendidas en su totalidad.
Por otra parte, los estudiantes de la Institución Educativa República de Israel, en un promedio de más de la mitad, consignan que las acciones se están desarrollando de manera positiva. En cuanto a la Institución Educativa Santa Isabel de Hungría sede Llano Verde Invicali, se demuestra que está en un promedio superior a la mitad, los resultados indican que lo efectuado se está desarrollando acorde a las necesidades de los estudiantes.

Ahora bien, desde una perspectiva general que implica la mirada del observador, las entrevistas al coordinador, docentes y estudiantes y la coherencia en sus discursos se exteriorizan en las acciones que se desarrollan en las IE ilustradas en la tabla 3.

Tabla 3. Acciones incluyentes

\begin{tabular}{|c|c|c|c|}
\hline Acciones & $\begin{array}{l}\text { Institución Educativa } \\
\text { Santa Isabel de Hungría } \\
\text { sede Alfonso López }\end{array}$ & $\begin{array}{l}\text { Institución Educativa } \\
\text { Santa Isabel de Hungría } \\
\text { Llano Verde Invicali }\end{array}$ & $\begin{array}{l}\text { Institución Educativa } \\
\text { República de Israel }\end{array}$ \\
\hline $\begin{array}{l}\text { Acceso y la permanencia de los } \\
\text { estudiantes }\end{array}$ & $\mathrm{x}$ & $\mathrm{x}$ & $\mathrm{x}$ \\
\hline $\begin{array}{l}\text { Seguimiento individualizado de } \\
\text { casos especiales }\end{array}$ & $\mathrm{x}$ & $\mathrm{x}$ & $\mathrm{x}$ \\
\hline $\begin{array}{l}\text { Reconocimiento de los grupos de } \\
\text { poblaciones }\end{array}$ & $\mathrm{x}$ & $\mathrm{x}$ & $\mathrm{x}$ \\
\hline Ajustes en casos especiales & $\mathrm{x}$ & $\mathrm{x}$ & $\mathrm{x}$ \\
\hline $\begin{array}{l}\text { Participación de todos los } \\
\text { miembros de la comunidad en las } \\
\text { diversas actividades }\end{array}$ & $\mathrm{x}$ & $\mathrm{x}$ & $\mathrm{x}$ \\
\hline $\begin{array}{l}\text { Desarrollo de propuestas } \\
\text { colaborativas entre los estudiantes }\end{array}$ & $\mathrm{x}$ & $\mathrm{x}$ & $\mathrm{x}$ \\
\hline $\begin{array}{l}\text { Pertinencia con respecto a las } \\
\text { necesidades de los estudiantes }\end{array}$ & $\mathrm{x}$ & $\mathrm{x}$ & $\mathrm{x}$ \\
\hline $\begin{array}{l}\text { Discurso inclusivo en ciertas } \\
\text { situaciones del aula }\end{array}$ & $\mathrm{x}$ & $\mathrm{x}$ & $\mathrm{x}$ \\
\hline $\begin{array}{l}\text { Adaptaciones realizadas en los } \\
\text { planes de clase y efectuadas en } \\
\text { aula }\end{array}$ & $\mathrm{x}$ & $\mathrm{x}$ & $\mathrm{x}$ \\
\hline Formaciones en inclusión & $\mathrm{x}$ & $\mathrm{x}$ & $\mathrm{x}$ \\
\hline $\begin{array}{l}\text { Creación de un grupo } \\
\text { interdisciplinario que fomente } \\
\text { procesos de inclusión }\end{array}$ & $\mathrm{x}$ & $\mathrm{x}$ & $\mathrm{x}$ \\
\hline
\end{tabular}

Fuente: elaboración propia 
La información se extrae y se propone como elementos positivos encontrados y valorados significativamente por todos los miembros de la comunidad, y a su vez adoptados como aportes para las prácticas educativas inclusivas institucionales, comprendidas desde lo que menciona la Organización de Estados Iberoamericanos para la Educación, la Ciencia y la Cultura (OEI) - OEI (2009): "No hay buenas prácticas ideales, sino que dependen del contexto en el que se desarrollan". Por consiguiente, desde su entramado conceptual y constructivo, las prácticas determinan los procesos de selección de actividades, las cuales visualizan diferentes momentos formativos, métodos y recursos que evidencian la intencionalidad primaria de contribuir en la adquisición de conocimientos. Sin embargo, el direccionamiento que plantea el Ministerio de Educación Nacional (MEN) no es coherente con las necesidades contextuales institucionales ya que los cambios a realizar están en proceso y la directriz en algunos casos no se muestra acorde con todas las necesidades de la región.

En otras palabras, se indica que las instituciones están en proceso de prácticas educativas inclusivas y las características contextuales influyen directamente en las acciones que esperan los estudiantes y en cada una de ellas se están efectuando de acuerdo con lo que consideran que deberían hacer.

\section{Elementos emergentes en las prácticas educativas inclusivas}

En la intervención realizada con los miembros de las comunidadades educativas se identificó que en estos espacios se construyen ele- mentos de apoyo e interacción relacionados con las prácticas educativas inclusivas, para ello se resalta que, en las entrevistas realizadas a los maestros, estos comentaron de manera especial que su mayor conocimiento para fortalecer estas habilidades en la construcción de una educación inclusiva lo han adquirido en la dinámica que desarrollan con los estudiantes, puesto que son ellos quienes están de manera continua conociendo sus habilidades, barreras, gustos y situaciones complejas, llevan a los docentes a explorar, analizar y crear intervenciones que den apoyo a estas situaciones diversas que emergen en cada uno de los estudiantes y que necesitan respuestas adecuadas para ayudar en su desarrollo de vida.

Por otra parte, El diseño curricular desde las realidades académicas, marcos referenciales y normativos corresponde a apuestas a nivel político que, en la mayoría de los casos se encuentran en debate social y se abordan desde políticas públicas para dar respuestas a coyunturas sociales, las cuales recaen sobre los lineamientos nacionales, municipales y finalmente institucionales. En este caso, el diseño curricular es elemento fundamental en los procesos de las prácticas educativas inclusivas y en las tres Instituciones Educativas se confirma desde una revisión documental que se encaminan a ser coherentes con el Proyecto Educativo Institucional (PEI) y que los planes de área, al igual que los de clase, se constituyen de la misma manera. Para verbigracia, se realiza encuesta y observación sobre cómo los estudiantes perciben la flexibilidad del diseño curricular, con los siguientes resultados: 
Tabla 4. Diseño curricular

\begin{tabular}{|c|c|c|c|}
\hline Ítems & $\begin{array}{l}\text { Institución Educativa } \\
\text { Santa Isabel de Hungría } \\
\text { sede Alfonso López (\%) }\end{array}$ & $\begin{array}{c}\text { Institución Educativa } \\
\text { Santa Isabel de Hungría } \\
\text { sede Llano Verde Invicali } \\
\text { (\%) }\end{array}$ & $\begin{array}{c}\text { Institución Educativa } \\
\text { República de Israel } \\
\text { (\%) }\end{array}$ \\
\hline $\begin{array}{l}\text { Reconozco que mi profesor organiza } \\
\text { la clase de acuerdo con las necesi- } \\
\text { dades y capacidades que los estu- } \\
\text { diantes tenemos. }\end{array}$ & 31 & 45 & 74 \\
\hline $\begin{array}{l}\text { La institución y mi profesor se preo- } \\
\text { cupan por mi aprendizaje y hacen } \\
\text { algunos cambios y mis familiares son } \\
\text { informados. }\end{array}$ & 51 & 51 & 80 \\
\hline $\begin{array}{l}\text { En las evaluaciones todos participa- } \\
\text { mos y en algunas de ellas se realizan } \\
\text { cambios de acuerdo con lo que cada } \\
\text { uno de nosotros podemos responder. }\end{array}$ & 25 & 64 & 59 \\
\hline
\end{tabular}

Fuente: elaboración propia

A partir del ejercicio de trazabilidad realizado en los procesos de observación en el aula y las expresiones de los estudiantes se percibe lo mencionado en la preparación de las clases $\mathrm{y}$ en las adaptaciones hechas para algunos estudiantes que las requieren, así mismo para las evaluaciones. Aunque la comprensión que conciben los estudiantes, en especial en la Institución Educativa Santa Isabel de Hungría sede Alfonso López y sede Llano Verde Invicali, no alcanza a demostrar que los maestros realizan los ajustes de tal manera que, aunque se hagan las mismas actividades, la forma de plantear su desarrollo no es igual; en otras palabras, los estudiantes no perciben a profundidad los cambios que se realizan porque no comprenden la diferencia como lo hace el observador.

Es así como los diseños curriculares son permeados por las prácticas educativas inclusivas y en los tres casos expusieron ajustes realizados desde la planeación de clase, su desarrollo, evaluación e informe por periodo o boletín. Dicho de otra manera, el paso a paso que realizan las instituciones apoyadas en documentos, entre los cuales resalta el Plan Individual de Ajustes Razonables (Piar) establecido por el Ministerio de Educación Nacional y adaptado por cada una de las instituciones para replantear las dinámicas que se establecen en el aula, aciertan en la creación de otros documentos que permiten realizar procesos individualizados que favorecen los avances de cada estudiante.

En concordancia, se afirma que lo realizado se muestra acorde con las prácticas educativas inclusivas porque, tal como lo expresan Durán, et al. (2005), los cambios que se realicen son imprescindibles en pro de establecer escuelas inclusivas capaces de enseñar en diferentes contextos y realidades heterogéneas, dispuestas a contemplar conocimientos teóricos y prácticos sobre las necesidades educativas más significativas que se encuentren asociadas a la diversidad social, que es la situación que se presenta en cada uno de los casos.

Por lo anterior, se considera como una ruta que permite el reconocimiento de la diversidad de los sujetos, que despierta una acción de acompañamiento y revisión constante en los avances que se realizan con cada uno de ellos. Sin embargo, mencionan tanto las instituciones como los docentes que todavía están en proceso 
de construcción ya que cada día aparecen nuevos retos, como por ejemplo, "de aquel menor de trece años que debe estar vinculado a un colegio para que la policía no se lo lleve porque es menor infractor en contra de su voluntad"; de esta misma manera, todos manifestaron que aunque se haga el proceso en las Instituciones Educativas, la cantidad de estudiantes y apoyos interdisciplinarios para algunos casos resulta primordial para la calidad educativa, por tal motivo, continuamente llevan a repensar sobre la forma correcta para accionar sin excluir y ofrecer así la posibilidad de brindar una educación acorde con las necesidades de los estudiantes. De la misma forma, se cuestionan sobre la dinámica social que se presenta, ya que mencionan la ruptura del proceso académico porque al finalizar bachillerato, no existe variedad de opciones para continuar con lo edificado.

No obstante, demuestran lo evidenciado en la información recolectada, otro elemento con preocupación con respecto a las pruebas externas dado que, aunque se dé atención a la diversidad y se realicen los ajustes correspondientes para apoyar académicamente a los estudiantes, solamente se hace de forma excepcional para estudiantes con discapacidad cognitiva y no se tienen presentes los factores sociales particulares (estudiantes desplazados, desnutridos, bajo la responsabilidad de madres sustitutas, pos conflicto, entre otros) que presentan otras dinámicas y requieren un acompañamiento especial en pro de evidenciar un desempeño escolar adecuado para la evaluación nacional.

Otro elemento que se presenta es el dispositivo didáctico con respecto al cual se indagó a profundidad sobre algún elemento que fuera significativo, en los tres casos hay coincidencia al afirmar que hay propuestas de diversas maneras de aprender, pero no señalaron alguna en especial. En este sentido, se interrogó a los maestros y todos corroboraron lo mencionado por los estudiantes y afirmaron que los que habían realizado los habían aprendido en formaciones y los habían modificado de acuerdo con las necesidades de los estudiantes. Para corroborar la información se retoma la encuesta realizada a los estudiantes:

Tabla 5. Uso de dispositivos didácticos

\begin{tabular}{|c|c|c|c|}
\hline Ítems & $\begin{array}{l}\text { Institución Educativa } \\
\text { Santa Isabel de } \\
\text { Hungría sede Alfonso } \\
\text { López (\%) }\end{array}$ & $\begin{array}{c}\text { Institución Educativa } \\
\text { Santa Isabel de Hungría } \\
\text { sede Llano Verde Invicali } \\
(\%)\end{array}$ & $\begin{array}{l}\text { Institución Educativa } \\
\text { República de Israel } \\
(\%)\end{array}$ \\
\hline $\begin{array}{l}\text { Le entiendo a mi profesor los temas } \\
\text { que explica porque lo hace con ejem- } \\
\text { plos, imágenes y otras ayudas. }\end{array}$ & 51 & 60 & 78 \\
\hline $\begin{array}{l}\text { El docente hace uso de diverso } \\
\text { tipo de material para que todos los } \\
\text { estudiantes puedan aprender con } \\
\text { facilidad. }\end{array}$ & 71 & 50 & 67 \\
\hline $\begin{array}{l}\text { Mi profesor realiza también las acti- } \\
\text { vidades que se deben llevar a cabo en } \\
\text { grupo. }\end{array}$ & 39 & 93 & $67 \%$ \\
\hline
\end{tabular}


En este sentido, las formaciones, creaciones y adaptaciones que se realicen al dispositivo didáctico deberán ser repensadas constantemente desde la función que cumplen, las intencionalidades pedagógicas, sociales y políticas -entre otrasque convergen como excusas para el aprendizaje, ya que los resultados porcentuales asignados a los maestros indican que hacen uso de diversas formas para enseñar. Una de ellas se enfoca en desarrollar todo ello mediante un trabajo colaborativo -aunque en uno de los casos no se realicen- los estudiantes refieren que son las que más les gustan porque se sienten más incluidos y consideran que aprenden con más facilidad, ya que los compañeros pares también permiten compartir el conocimiento.

De allí se determina que los espacios de las instituciones educativas están ligados directamente a las estrategias que los maestros desarrollen en la clase; así mismo, a los instrumentos que retomen o diseñen como vínculo significativo que entreteje el conocimiento, los aprendizajes y las relaciones que encaminan la interacción como ejercicio primordial en el desarrollo intelectual y en la formación de la personalidad de los estudiantes.

En los procesos de formación académica (pregrado) los maestros no cursaron asignaturas que visualizaran la diversidad de los sujetos en las aulas de clase y han adquirido aprendizajes debido a situaciones especiales experimentadas en casos puntuales en los que desarrollan estrategias para la atención inmediata. No obstante, mencionan que a la fecha no se piensa, por ejemplo, en otras características que presentan los estudiantes (con familias disfuncionales, víctimas del conflicto, menores trabajadores, post conflicto, etc.).

El desarrollo de trabajo colaborativo es esencial para los estudiantes ya que consideran que la interacción con sus pares apoya el aprendizaje y genera aceptación frente a las formas de adquisición del conocimiento y de ser de los sujetos. Sin embargo, aunque se reconozca como estrategia motivadora, para los estudiantes, en ocasiones no es incluyente porque discrimina al momento de revisar los resultados individuales que se basan en una evaluación por competencias.

Lo mencionado debería vincular de manera directa a los docentes, administrativos, padres de familia, estudiantes y agentes sociales en un trabajo preciso que convoque a un ejercicio colaborativo y con la intencionalidad que plantea Fielding (1999), como medio para alcanzar de manera conjunta replanteamientos sobre los intereses comunes, metas propias, políticas y dinámicas educativas con relación a las diversas situaciones que se presentan en los contextos escolares.

Lo suministrado permite recalcar que el resultado que presenta cada una de las instituciones determina que, aunque tengan el mismo marco normativo, las dinámicas y concepciones de los procesos de formación -tanto de directivas, docentes y estudiantes del mismo municipio- son a veces disímiles. En procesos de observación hay coherencia con lo que se expresa y dichas dinámicas y concepciones de los procesos de formación son determinantes; por ello es esencial continuar con la atención de estudiantes de naturaleza heterogénea en sus formas de aprender y en palabras de Arnaiz (2007), brindar una formación continua al profesorado y estar atentos a la actitud del docente con respecto a la creación de instrumentos que contribuyan al éxito o fracaso en la atención a la diversidad.

\section{Conclusiones}

La inclusión es vista por los directivos y maestros de las Instituciones Educativas como la posibilidad que tienen los estudiantes con barreras (de aprendizaje, sociales, culturales, económicas, físicas, entre otras) para acceder a la educación, con mayor énfasis en alumnos con algún tipo de discapacidad, dado que consideran que estos presentan un nivel de complejidad diferente con respecto a las atencio- 
nes a brindar. En cambio, los estudiantes la comprenden como el derecho que tienen para acceder a la educación, el cual debe ser justo para la totalidad y diversidad de estudiantes que asisten.

Desde las coordinaciones, el direccionamiento se funda en el marco legal y a su vez, en las orientaciones que brindan a los maestros a su cargo. Se resalta que las instituciones educativas abordadas fomentan desde el discurso: planear, ejecutar y evaluar constantemente a los estudiantes en la diversidad, hacen seguimiento y están enterados de cada uno los casos particulares de manera integral, pero se habla desde la acreditación que hace el MEN y no desde los saberes que tiene el maestro en todo su proceso de formación. Moliner (2008) considera este aspecto fundamental, ya que es ver cómo se transforma el sistema educativo para responder a la diversidad, revisar el proceso y los respectivos ajustes para encaminarla a una política pública inclusiva desde el direccionamiento.

En cuanto a las prácticas educativas inclusivas, algunas son orientadas por las directrices de la comunidad educativa, otras desde la experiencia del profesor y las demás, a partir de la interacción con los estudiantes que presentan alguna situación que reta al docente a repensar su rol como maestro. Por parte de los estudiantes, se afirma que, aunque los estudiantes no consideren las acciones desarrolladas como incluyentes en su discurso, las evidencias demuestran lo contrario. De allí que lo expresado por los participantes asevera que las acciones que realizan los docentes no corresponden a acciones inmediatas o únicas en el proceso de la verificación de la clase, pero a la vez, ellos reconocen que, a la fecha, son coherentes con respecto al tiempo compartido en la comunidad académica. Por tal motivo, son acordes a las necesidades de las realidades y son prácticas incluyentes que están en continuo movimiento.

Los resultados demuestran que, aunque se realicen los procesos, no siempre se deben determinar por unos logros ya completamente obtenidos, tal como expresa Ainscow Mel (2003), ya que la inclusión está encaminada a una búsqueda interminable de formas acordes a los sujetos para responder a la diversidad; por lo tanto, se enfoca en aprender a vivir constantemente con la diferencia y al tiempo, aprender de ella de forma continua.

Los maestros reconocen que la inclusión y las prácticas educativas inclusivas no tienen en el aula una clase única e inamovible, ya que consideran que estas se deben reconstruir con el tiempo y los estudiantes las deben evaluar constantemente de tal forma que las percepciones que tienen sobre el reconocimiento de la diversidad de sujetos, su trato y la forma en que se relacionan con los miembros de la comunidad sean determinantes en las prácticas. Se suma allí que los aprendizajes que tienen los estudiantes no evidencian igualdad, ya que la cantidad de ellos no permite enfocar con detalle la atención y que esta sea más personalizada, es así como lo manifiesta la UNESCO (2004) que afirma que esto influye en los logros del aprendizaje y por ende, en las trayectorias educativas.

\section{Recomendaciones}

A nivel global se considera que las prácticas educativas inclusivas se podrán visibilizar con más precisión al determinar que existan políticas acordes con las necesidades contextuales, esto permitirá que en el ambiente escolar se dinamicen acciones y que estas sean reconocidas como parte de una cultura que incentiva el reconocimiento a la otredad como una posibilidad y no precisamente como sujetos que se miden por su conocimiento.

El sistema educativo se debe direccionar con base en un marco nacional y local que permita comprender que las políticas, prácticas y cultura requieren ser repensadas como indicadores primarios que deben ir mejorando en cualquier contexto educativo y que solo se pueden ver como 
dimensiones que conllevan adaptaciones acordes con una calidad educativa.

Fomentar la identidad de cada uno de los sujetos es necesario, ya que se piensa el currículo en tanto totalidad, pero en cierta medida se desconocen las prácticas educativas inclusivas que hacen que los estudiantes se sientan en las mismas condiciones con respecto a sus prácticas culturales y las formas de percibir las situaciones que se desarrollan en el medio escolar.

\section{Referencias}

Ainscow, M. (2003). Desarrollo de sistemas educativos Inclusivos. Ponencia en el congreso La respuesta a las necesidades educativas especiales en una escuela vasca inclusiva. San Sebastián, España. http://lupitahdt.galeon.com/index_ archivos/800/p6.pdf

Arnaiz, P. (2007). Como promover prácticas inclusivas en Educación Secundaria. Revista perspectiva de los centros de profesorado en Andalucía, 1(14), 57-71.

Blanco, R. (2014). Avances y desafíos de la Educación Inclusiva en Iberomérica. Organización de los Estados Americanos.

Booth, T., y Ainscow, M. (2015). Guía para la educación inclusiva. Desarrollando el aprendizaje y la participación en los centros escolares. Organización de los Estados Americanos para la Educación y la Ciencia.

Durán, D., Echeita, G., Climent, G., Miquel, E., Ruiz, C., y Sandoval, M. (2005). Primeras experiencias de uso de la Guía para la evaluación y mejora de la Educación Inclusiva (Index for
Inclusión) en el Estado Español. REICE, Revista Electrónica Iberoamericana sobre Calidad, Eficacia y Cambio en Educación, 3(1), 464-467. https://www. redalyc.org/pdf/551/55130145.pdf

Fielding, M. (1999). Radical collegiality: affirming teaching as an inclusive professional practice. Australian Educational Researcher, 26(2), 1-34.

Moliner, O. (2008). Condiciones, procesos y circunstancias que permiten avanzar hacia la inclusión educativa: retomando las aportaciones de la experiencia canadiense. Revista Electrónica Iberoamericana sobre calidad, Eficacia y Cambio en Educación, 6(2). http:// www.rinace.net/arts/vol6num2/art3.pdf

Organización de las Naciones Unidas (ONU). (1948). Declaración Universal de los Derechos Humanos. ONU

Organización de Estados Iberoamericanos para la Educación, la Ciencia y la Cultura (OEI) (2009). Guía para para reflexión y valoración de las prácticas inclusivas. OEI.

Parrilla, A., y Susinos, T. (2004). El desafío de la educación inclusiva a las exclusiones en los sistemas y comunidades educativas. En J. López, M. Sánchez y P. Murillo (eds.), Cambiar con la sociedad, cambiar la sociedad. Actas del $8^{\circ}$ Congreso interuniversitario de Organización de Instituciones Educativas (pp. 195200). Universidad de Sevilla.

UNESCO (2004) Temario abierto sobre la Educación Inclusiva. Oficina Regional de Educación de la Unesco para América Latina y el Caribe Orealc / Unesco. http://unesdoc.unesco.org/ images/0012/001252/125237so.pdf

UNESCO (2005). Guidelines for inclusion: Ensuring Access to education for All. Unesco. 